\title{
TOLERANCIA Y ACUMULACIÓN DEL PLOMO EN BACCHARIS PENTLANDII DC SEGÚN TRATAMIENTO DE SUBSTRATOS CON RELAVES MINEROS
}

\section{TOLERANCE AND ACCUMULATION OF LEAD IN BACCHARIS PENTLANDII DC AS TREATMENT MINING TAILINGS SUBSTRATES}

\author{
Edith Orellana Mendoza \\ Facultad de Ciencias Forestales y del Ambiente, Universidad Nacional del Centro del Perú.
}

\begin{abstract}
RESUMEN
El uso de especies vegetales como agentes descontaminantes de suelos contaminados por metales pesados constituye una alternativa tecnológica económica y limpia que merece ser estudiada. El estudio consiste en determinar la capacidad de tolerancia y acumulación del plomo en Baccharis pentlandii DC según tratamiento de substratos con relaves mineros, las unidades experimentales estudiadas correspondieron a plántulas de $3.5 \mathrm{~cm}$ de alto, las que son establecidas en bolsas de polietileno conteniendo substratos con mezcla de relaves mineros; se adopta el diseño completamente al azar con tres tratamientos y seis repeticiones. A los diez meses, $B$. pentlandii registra diferencias en la tolerancia y acumulación del $\mathrm{Pb}$, las plantas experimentan crecimientos diferentes y significativos. La capacidad de acumulación de plomo varía de acuerdo a la proporción de relave en el substrato; tratamientos en la proporción 1(suelo):1(relave) y 2 (suelo):1(relave) influyen en la mayor acumulación en la raíz respecto a la parte aérea de la planta. La mayor concentración total de Pb se observa en el substrato de suelo agrícola tratado con relave en la proporción 1:1 con $400 \mathrm{mg} \mathrm{kg}^{-1}$ de Pb. De acuerdo a los valores de acumulación esta especie no se puede clasificar como hiperacumuladora de plomo.
\end{abstract}

Palabras clave: Fitorremediación, metales pesados, tolerancia, acumulación

\begin{abstract}
The use of plant species such as decontamination agents of contaminated soils with heavy metals is an economical and clean technological alternative that deserves to be studied. The study consists of determining the ability of tolerance and accumulation of lead in Baccharis pentlandii DC according to treatment of substrates with tailings. Studied experimental units corresponded to seedlings of $3.5 \mathrm{~cm}$ high, which are established in polyethylene bags containing substrates with mixture of tailings; the completely randomized with three treatments and six replications design is adopted. At ten months, B. pentlandii records differences in tolerance and accumulation of $\mathrm{Pb}$, plants experience different and significant growths. The lead storage capacity varies according to the proportion of tailings in the substrate; treatments in the ratio 1 (soil): 1 (tailings) and 2 (floor): 1 (tailings) influence the greater accumulation in the root on the aerial part of the plant. The highest total concentration of $\mathrm{Pb}$ is observed in the substrate of agricultural soil treated with tailings in the proportion 1:1 with $400 \mathrm{mg} \mathrm{kg} \mathrm{-} 1$ of Pb. According to the values of accumulation this species cannot be classified as lead hiperacumulator.
\end{abstract}

Keywords: Phytoremediation, heavy metal, tolerance, accumulation 


\section{INTRODUCCIÓN}

En la actualidad, la situación en la región Junín es bastante preocupante, lo cual se debe a la creciente contaminación del agua y el suelo, causada por los relaves mineros. En esta zona del país existen un $15 \%$ de relaves mineros post operativos, (Ministerio de Energía y Minas [MEM], 2007), los cuales contienen sustancias tóxicas como arsénico, cadmio, cianuro, cobre, mercurio, selenio, zinc, plomo, que, al tener contacto con el agua y el subsuelo, altera su composición natural, y esto, a su vez, afecta a la fauna, flora y población humana con riesgos a la salud.

La mayoría de las plantas no crecen en estos sitios, ya que las altas concentraciones de estos elementos son tóxicas para sus organismos; sin embargo, existe un grupo de plantas denominadas metalofitas, que son capaces de desarrollarse en estas condiciones. En las últimas décadas del siglo XX surgieron tecnologías basadas en el empleo de organismos vivos para descontaminar suelos o emplazamientos contaminados y recuperar los ecosistemas afectados. Cuando estas tecnologías se basan en el uso de plantas, globalmente reciben el nombre de fitorremediación (Salt et al., 1998), citado por Carpena y Bernal (2007). El concepto de usar plantas para limpiar suelos contaminados no es nuevo, desde hace cientos de años las plantas fueron propuestas para el uso en el tratamiento de aguas residuales. La fitorremediación de suelos contaminados se basa en el uso conjunto de plantas, enmiendas del suelo y técnicas agronómicas para eliminar, retener, o disminuir la toxicidad de los contaminantes del suelo (Ginocchio, 2004).

La investigación tuvo como propósito encontrar estrategias basadas en el uso de plantas que tienen la propiedad de acumular metales pesados; proceso denominado "fitorremediación", y de esta forma experimentar en condiciones naturales a fin de reducir la contaminación por metales pesados en suelos circundantes a pasivos ambientales. El objetivo del estudio fue determinar la tolerancia y acumulación del plomo en Baccharis pentlandii DC según tratamiento de substratos con relaves mineros.

\section{MATERIAL Y MÉTODOS}

El estudio se llevó a cabo en San Carlos, Huancayo -Junín. Se utilizaron bolsas de polietileno conteniendo substrato de suelo agrícola con relave minero conteniendo $\mathrm{Pb}$.

Las plantas de B. pentlandii se seleccionaron con pan de tierra del terreno de la Comunidad Campesina de Cullpa Alta con altura promedio de $3,5 \mathrm{~cm}$ de alto., y el relave minero del pasivo ambiental de la ex planta metalúrgica de Yauris - UNCP.

El experimento se condujo de acuerdo con el diseño completamente al azar con cuatro tratamientos (substrato con relave minero) y seis repeticiones, cada unidad experimental fue representada por una planta por repetición; los substratos con relaves mineros se prepararon según distribución de tratamientos, consistió en mezclar el suelo agrícola con el relave en las proporciones 2:1 (T2), 1:1 (T3) y 1: 2 (T4) y el substrato control (T1). La muestra estuvo constituida por 24 unidades experimentales

La recolección de datos se realizó mediante la observación directa e indirecta, utilizando como instrumentos una guía de observación, ficha de registro y el espectrofotómetro UV.

La variable principal de estudio fue la acumulación del $\mathrm{Pb}$ en el tejido vegetal, lo que permitió calcular el potencial efectivo en la parte aérea, raíz y total (parte aérea + raíz) mediante la multiplicación de peso seco de cada parte del tejido por la concentración de Pb en la misma. La distribución de Pb en la planta se hizo mediante una comparación de los porcentajes de plomo acumulado en la parte aérea de las plantas y en las raíces, con respecto a la acumulación total.

La evaluación de la tolerancia de las plantas a la acción del plomo, se midió a través del crecimiento y sobrevivencia a los diez meses de instalado el experimento. Para la medición de los niveles de concentración del plomo, se extrajeron al azar 2 muestras de la parte aérea y 2 de la parte radicular de cada tratamiento; a continuación se procedió al lavado con agua corriente y secado al horno a una temperatura de $75^{\circ} \mathrm{C}$, hasta peso constante. Se pesaron por separado la raíz de la parte aérea en balanza electrónica. La suma de la raíz y parte aérea se consideró como el peso seco total de la materia seca.

A continuación se procedió al proceso de mineralización de las muestras, el cual consistió en triturar las hojas y tallos y las raíces secas por separado con ayuda del mortero, luego se pesó 4,0 g de cada 
muestra experimental y se colocó en crisoles de porcelana debidamente enumerados. Seguidamente las muestras en los respectivos crisoles se llevaron a la estufa eléctrica con asbesto durante 2 a 3 horas hasta lograr el carbonizado de las muestras. Los que posteriormente se llevaron a la mufla durante 3 horas a una temperatura de $600^{\circ} \mathrm{C}$.

Una vez mineralizadas las muestras de la parte aérea y radicular, fueron llevadas al Laboratorio de Análisis Químico de la Facultad de Ingeniería Química de la Universidad Nacional del Centro del Perú, para su análisis y determinación del contenido de plomo absorbido por las plantas de B. pentlandii. Para comparar la acumulación de $\mathrm{Pb}$ en la planta según tratamiento de substratos con relave, se realizó el análisis de varianza, mediante el software SPSS, la comparación de medias se hizo mediante la prueba de Tukey $p<=0,05$ ).

\section{RESULTADOS}

\section{Tolerancia al plomo}

Tabla 01: Análisis de varianza de altura a los diez meses

\begin{tabular}{lcccc}
\hline \multicolumn{1}{c}{ F.V. } & S.C & gl & CM & $F_{\mathrm{C}}$ \\
\hline Tratamientos & 1123.78 & 2 & 561.89 & $33.57^{*}$ \\
Error & 234.33 & 14 & 16.73 & \\
Total & 1358.11 & 16 & & \\
\hline
\end{tabular}

Tabla 02: Comparación de medias de crecimiento en altura

\begin{tabular}{lll}
\hline \multicolumn{1}{c}{ Substratos con relaves } & N & Altura (cm) \\
\hline Testigo (T1) & 6 & $16.50 \mathrm{a}$ \\
Substrato 1:1 (T3) & 5 & $27,00 \mathrm{~b}$ \\
Substrato 2:1 (T2) & 6 & $35,83 \mathrm{c}$ \\
\hline
\end{tabular}

Tabla 03. Análisis de varianza de materia seca parte aérea y raíz de $B$. pentlandii

\begin{tabular}{lcccc}
\hline \multicolumn{1}{c}{ F.V. } & S.C. & gl & CM & $\mathrm{F}_{\mathrm{c}}$ \\
\hline Tratamientos & 4,20 & 2 & 2,10 & $15,56^{*}$ \\
Error & 0,40 & 3 & 0,13 & \\
Total & 4,60 & 5 & & \\
\hline Tratamientos & 0,97 & 2 & 0,48 & $6,46 \mathrm{~ns}$ \\
Error & 0,22 & 3 & 0,07 & \\
Total & 1,19 & 5 & & \\
\hline
\end{tabular}

Tabla 04: Comparación de medias de producción de materia seca parte aérea

\begin{tabular}{llc}
\hline Substratos con relaves & $N$ & $\begin{array}{c}\text { Producción de materia } \\
\text { seca }(g)\end{array}$ \\
\hline Testigo (T1) & 2 & $7,20 \mathrm{a}$ \\
Substrato 1:1 (T3) & 2 & $8,95 \mathrm{~b}^{\star}$ \\
Substrato 2:1 (T2) & 2 & $9,00 \mathrm{~b}^{\star}$ \\
\hline
\end{tabular}

${ }^{*}$ Medias con letras iguales son estadisticamente iguales (Tukey 0,05)

\section{Acumulación de $\mathrm{Pb}$ en tejido vegetal}

Tabla 05: Análisis de varianza de acumulación de Pb parte aérea y raíz

\begin{tabular}{lcccc}
\hline \multicolumn{1}{c}{ F.V. } & S.C. & gl & C.M. & $F_{c}$ \\
\hline Tratamientos & 4684,64 & 2 & 2342,32 & $49,67^{*}$ \\
Error & 141,44 & 3 & 47,14 & \\
Total & 4826,09 & 5 & & \\
\hline Tratamientos & 17474,45 & 2 & 8737,22 & $12,76^{*}$ \\
Error & 2053,35 & 3 & 684,45 & \\
Total & 19527,80 & 5 & & \\
\hline
\end{tabular}

Tabla 06: Comparación de medias de acumulación de Pb parte aérea y raíz

\begin{tabular}{lccc}
\hline \multirow{2}{*}{ Substrato con relaves } & & \multicolumn{2}{c}{$\begin{array}{c}\text { Acumulación de Pb mg kg-1 de } \\
\text { materia seca }\end{array}$} \\
\cline { 2 - 4 } & $N$ & Parte aérea & Raíz \\
\hline Testigo (T1) & 2 & $0,81 \mathrm{a}$ & $3,11 \mathrm{a}$ \\
Substrato 2:1 (T2) & 2 & $57,41 \mathrm{~b}$ & $59,05 \mathrm{~b}$ \\
Substrato 1:1 (T3) & 2 & $62,43 \mathrm{~b}$ & $134,80 \mathrm{~b}$ \\
\hline
\end{tabular}

Fig. 01: Distribución de Pb acumulado (\%) parte aérea y raíz de B. pentlandii

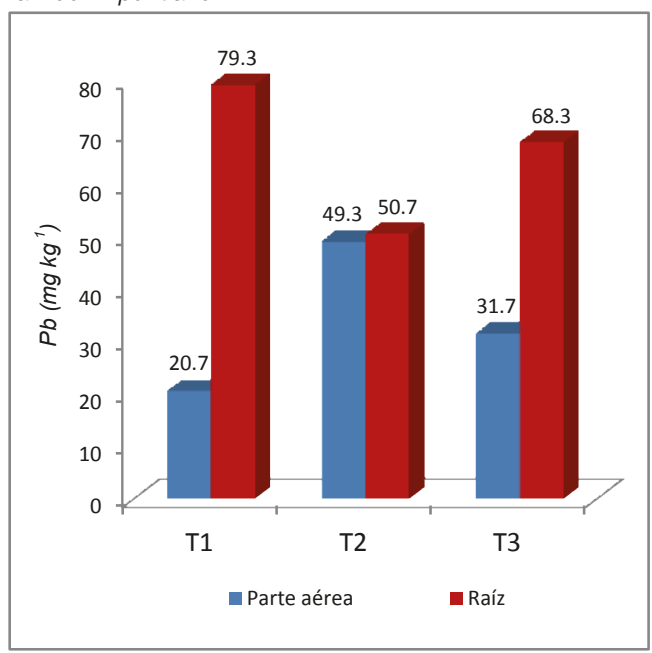


Fig. 02.: Acumulación total de $P b\left(\mathrm{mg} \mathrm{kg}^{-1}\right)$ parte aérea + raíz en B. pentlandii

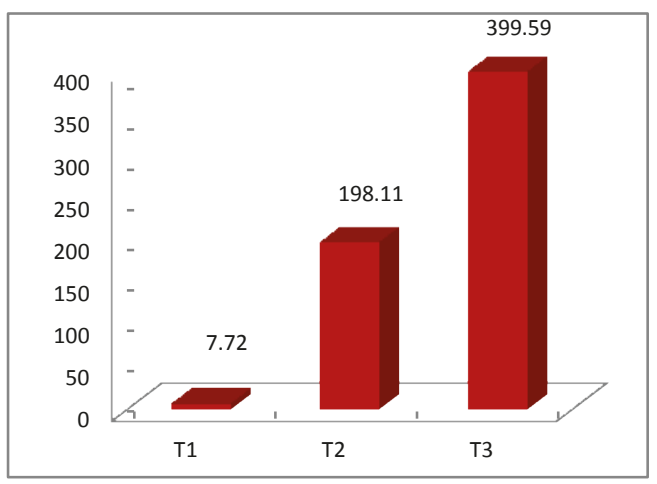

\section{DISCUSIÓN}

\section{Tolerancia al plomo}

El crecimiento en altura de B. pentlandii, fue diferente y significativa, a los 10 meses registraron mayores crecimientos en substratos en la proporción 2 (suelo agrícola) y 1 (relave) con 34,0 y 36,0 cm respectivamente en comparación al substrato en proporción 1 (suelo) : 1 (relave) (Tabla 01 y 02), mostrando las plantas tolerancia al plomo y otros metales pesados que contiene el relave; a los diez meses el crecimiento apical se fue haciendo más lento, observándose defoliación en la parte basal de la planta y crecimiento de nuevos brotes laterales. Sobrevivencias de $100 \%$ de plantas corresponden al tratamiento control y al tratamiento de suelo agrícola en la proporción 2:1 y de 83\% a plantas en substratos en la proporción 1:1; substratos con 25\% de suelo agrícola y $75 \%$ de relave (1:2), resultaron tóxicos para las células de las plantas, éstas registraron un $100 \%$ de mortandad a los 15 días de establecido, no toleran contenidos altos de plomo (superiores a 2500 mg Pb kg-1). Los resultados corroboran que aquellos metales que, siendo elementos pesados como el plomo, son "tóxicos" para la célula; sin embargo, cualquier elemento que a priori es beneficioso para la célula, en concentraciones excesivas puede llegar a ser tóxico (Navarro, Aguilar y López, 2007).

Según lo reportado por Kabata-Pendias (2000) y Rodríguez et al. (2006), el plomo puede causar diversos daños a las plantas y en diferentes grados de acumulación. Los mecanismos de fitotoxicidad de $\mathrm{Pb}$ están relacionadas, con la permeabilidad de la membrana celular, reacciones de grupos sulfidrilos (-SH) con cationes y afinidad para reaccionar con grupos fosfatos (Rodríguez et al., 2006). Asimismo la sensibilidad de las especies vegetales a los metales pesados varía considerablemente a través de reinos y familias, siendo las plantas vasculares ligeramente más tolerantes (Rosa et al., 1999) citado por Prieto et al. (2009).

Se detectaron diferencias significativas en la producción de materia seca en la parte aérea de la planta (Tabla 03), unidades experimentales en substratos con relave en las proporciones 2:1 y 1:1 mostraron mayores producciones con 9,0 y 8,95 g de materia seca respectivamente (Tabla 04). No se observaron diferencias estadística significativa en el peso seco de la raíz, el substrato control y los tratamientos con relave no influyeron en la producción de materia seca de la raíz, el $\mathrm{Pb}$ reduce el crecimiento radicular y la frecuencia de células mitóticas y el incremento de la frecuencia de células aberrantes en A. cepa (Lerda, 1992). Todas las plantas absorben metales del suelo donde se encuentran pero en distinto grado, dependiendo de la especie vegetal, y de las características y contenido en metales del suelo. Además Prieto et al. (2009), señala que cuando el contenido de metales pesados en el suelo alcanzan niveles que rebasan los límites máximos permitidos causan efectos inmediatos como inhibición del crecimiento normal y el desarrollo de las plantas, y un disturbio funcional en otros componentes del ambiente así como la disminución de las poblaciones microbianas del suelo.

\section{Acumulación de plomo en el tejido vegetal}

La absorción y posterior acumulación dependen en primera instancia del movimiento de los metales desde la solución en el suelo a la raíz de la planta. Plantas de $B$. pentlandii absorbieron y acumularon plomo de manera diferente y significativa (Tabla 05). Mayores acumulaciones en la biomasa aérea se observaron en substratos con $50 \%$ de relave y $50 \%$ de suelo agrícola con $62 \mathrm{mg}$ de $\mathrm{Pb} \mathrm{kg}^{-1}$ y en substratos con $75 \%$ de suelo agrícola y $25 \%$ de relave con $57,4 \mathrm{mg} \mathrm{Pb} \mathrm{kg}^{-1}$ de materia seca.. 
La capacidad de acumulación de plomo en las raíces fue mayor y significativa en los tratamientos con relave minero en las proporciones 1:1 y 2:1 con 135 y 59 $\mathrm{mg} \mathrm{Pb} \mathrm{kg}{ }^{-1}$ de materia seca respectivamente (Tabla 06). En general las plantas presentaron una tendencia a acumular más plomo a medida que se incrementó la proporción de relave en el suelo, sin embargo plantas en proporciones de más de $50 \%$ de relave en el suelo no toleran y mueren; coincidiendo con lo reportado por Rodríguez et al. (2006). Según Kabata Pendias (2000) en algunas plantas el Pb es capaz de acumularse principalmente en las raíces siendo mínima su presencia en otras partes u órganos de los cultivos. Y la disponibilidad para las plantas está en función del pH del suelo; la mayoría de los metales tienden a estar más disponibles a pH ácido, excepto el As, Mo, Se y Cr, los cuales tienden a estar más disponibles a pH alcalino. La adsorción de los metales pesados está fuertemente condicionada por el pH del suelo..

De reporte del análisis químico de muestras, se observa acumulación de $\mathrm{Pb}$ en la materia seca de la parte aérea $\left(1,62\right.$ a $0.0 \mathrm{mg}$ de $\left.\mathrm{Pb} \mathrm{kg}{ }^{-1}\right)$ y raíces $(3,52$ y $2,7 \mathrm{mg}$ de $\mathrm{Pb} \mathrm{kg}{ }^{-1}$ ) en el substrato control, esto se explica en el sentido de que los metales pesados están presentes en el suelo como componentes naturales del mismo, o como consecuencia de las actividades antropogénicas (Prieto et al., 2009). Porta et al. (2003), al respecto, explica que la presencia de elementos traza en un medio se conoce como fondo geoquímico de un elemento, que consiste en la concentración del elemento en un determinado medio, en ausencia de cualquier aporte externo específico derivado de la actividad humana. El fondo geoquímico de un mismo elemento variará según el material originario, ya sea derivado de rocas ígneas o sedimentarias. El plomo, zinc y cadmio se hallan en la litósfera con unos contenidos medios (fondo geoquímico) de: $\mathrm{Cd}$ de $0,2 \mathrm{mg} \mathrm{kg}^{-1}, \mathrm{~Pb}$ de 16 $\mathrm{mg} \mathrm{kg}^{-1}$ y Zn de $80 \mathrm{mg} \mathrm{kg}^{-1}$, estos valores corresponden a los contenidos medios de las distintas rocas, tanto ígneas como sedimentarias. (Porta et al., 2003).

B. pentlandii acumuló más $\mathrm{Pb}$ en las raíces que en la parte aérea (Fig. 01), la mayor acumulación de Pb se logró en las raíces tanto en el substrato control como en los suelos tratados con relaves conteniendo $\mathrm{Pb}$ con 79,3 $\%$ (esto es, 3,11 mg de $\mathrm{Pb} \mathrm{Kg}^{-1}$ en su tejido radicular),
50,7 \% (59,05 mg de $\mathrm{Pb} \mathrm{Kg}^{-1}$ ) y 68,3\% (esto es 68,3 mg de $\mathrm{Pb} \mathrm{Kg}^{-1}$ ) discrepando con lo reportado por Rodríguez et al. (2006) quien encontró mayores concentraciones de $\mathrm{Pb}$ en la parte aérea 4 veces más que en la raíz en seis especies vegetales. Durán (2010) del mismo modo determinó que $B$. latifolia acumuló un mayor contenido de $\mathrm{Pb}$ en la parte aérea, 3,5 veces más respecto de la raíz. La acumulación de $\mathrm{Pb}$ en la parte aérea y radicular fue disminuyendo a medida que se incrementaba la proporción de relave con este elemento en el suelo; sin embargo no toleran elevadas dosis que superan los $2500 \mathrm{mg} \mathrm{de} \mathrm{Pb} \mathrm{kg}^{-1}$. Debido a que los factores que influyen en la movilización de metales pesados en el suelo son las características del suelo: pH, composición iónica de la solución del suelo, capacidad de cambio, presencia de carbonatos, materia orgánica, textura; naturaleza de la contaminación: origen de los metales y forma de deposición y condiciones medioambientales: acidificación, cambios en las condiciones redox, variación de temperatura y humedad (Prieto et al., 2009).

B. pentlandli no logró concentrar más de $1000 \mathrm{mg}$ de $\mathrm{Pb}$ $\mathrm{kg}^{-1}$ de materia seca, por lo que no puede considerarse hasta el momento como especie hiperacumuladora de $\mathrm{Pb}$, de acuerdo con el criterio establecido por Baker y Brooks (1989). La mayor concentración total de Pb alcanzó en el substrato de suelo agrícola tratado con relave en la proporción 1:1 con $400 \mathrm{mg} \mathrm{kg}^{-1}$ de $\mathrm{Pb}$ (Fig. 02). No coincidiendo con los resultados obtenidos por Durán (2010), B. latifolia acumuló más de 2500 $\mathrm{mg}-\mathrm{kg}^{-1}$ de $\mathrm{Pb}$, superando el valor límite para ser considerada como hiperacumuladora.

\section{CONCLUSIONES}

B. pentlandlii según tratamientos de relaves mineros con plomo $(\mathrm{Pb})$ experimentaron crecimientos diferentes y significativos, las unidades experimentales en tratamientos en la proporción 2:1 registraron sobrevivencias y alturas promedio superiores al testigo y al tratamiento en la proporción 1:1, mostrando tolerancia a la presencia del plomo en el substrato. Tratamientos en la proporción 1:1 y 2:1 influyeron en la mayor acumulación de plomo $(\mathrm{Pb})$ en la parte aérea y radicular de la especie. La mayor acumulación total se registró en la raíz de la planta. La mayor concentración total de $\mathrm{Pb}$ alcanzó en el substrato de suelo agrícola tratado con 
relave en la proporción 1:1 con $400 \mathrm{mg} \mathrm{kg}^{-1}$ de Pb. De acuerdo a los valores de acumulación esta especie no se puede clasificar como hiperacumuladora de plomo $(\mathrm{Pb})$.

\section{REFERENCIAS BIBLIOGRÁFICAS}

- Alkorta, I., Hernández - Allica, J., Becerril, J.M., Amezaga, I., Albizu, I. Garbisu, C. (2004). Recent finding on the phytoremediation of soils contaminated with environmentally toxic heavy metals and metalloids such as zinc, cadmium, lead and arsenic. Environmental Science Biotechnology, 3:71-90.

- Baker,A. y Brooks, R. (1989). Terrestrial higher plants which hyperaccumulate metallic elements: A review of their distribution, ecology and phytochemestry. Biorecovery, 1:81-108.

- Baker, A.J.M., McGrath, S.P., Reeves, R.D. and Smith, J.A.C. (2000). Metal hyperacumulator plants: a review of he ecology and physiology of a biological resource for phytoremediation of metal-polluted soils. En: hytoremediation of Contaminated Soil and Water (eds. Terry, N. y Bañuelos, G.), pp. 85-107, Lewis Publishers, Boca Raton, FL, SA.

- Becerril, J., Barrutia, J., García Plazaola, J., Hernández, A., Olano, J. y Garbisu, C. (2007). Especies nativas de suelos contaminados por metales: aspectos ecofisiológicos y su uso en fitorremediación. Ecosistemas, 16 (2).

- Brooks, R.R., Lee, J., Reeves, R.D. and Jaffré, T. (1977). Detection of nickeliferous rocks by analysis of herbarium specimens of indicator plants. Journal Geochemical Exploration, 7: 49-57.

- Calderón V. y Maldonado, M. (2008). Contaminación e intoxicación por plomo. México:Trillas.

- Carpena, R. y Bernal, M.(2007). Claves de la fitorremediación: fitotecnologías para la recuperación de suelos. España: Universidad Autónoma de Madrid.

- Donoso, C. (2001). Ecología forestal, el bosque y su medio ambiente. Facultad de Ciencias Forestales. ( $3^{a}$ ed.). Chile: Universidad Austral de Chile.

- Durán, P. (2010). Transferencia de metales de suelo a planta en áreas mineras: Ejemplos de los Andes peruanos y de la Cordillera Pre litoral Catalana. (Tesis doctoral). Universidad de Barcelona. Barcelona, España.

- ECOAMERICA. (2007). Fitoestabilización de relaves: Metales atrapados en una raíz. Recuperado de: www.ecoamerica.cl/mayo/2007.

- Hernández, R., Fernández, C. y Baptista, P. (2010). Metodología de la investigación. (5a ed.). México: Mc. Graw-Hill/Interamericana editores S.A.

- García, I. y Dorronsoro, C. (2005).Contaminación por metales pesados. En:Tecnología de Suelos. Universidad de Granada. Departamento de Edafología y Química Agrícola. Recuperado de htpp://edafología. ugr.es/conta/tema15/introd.htm

- Ginocchio, R. (2004). Metalofitas en América Latina: un recurso biológico y genético único y poco estudiado en la región. Revista Chilena de Historia Natural. 77: 185-194

- González, M., Muena, V., Cisternas, M. y Neaman, A. (2008). Acumulación de cobre en una comunidad vegetal afectada por contaminación minera en el valle de Puchuncaví, Chile Central. Revista Chilena de Historia Natural. 81: 279-291.

- Kabata-Pendias, A. (2000). Trace elements in soils and plants. ( $3^{\mathrm{a}}$ ed.). USA: CRC Press.

- Lerda, D. 1992. The effect of lead on Allium cepa L. Mutation Research, 281:89-92.

- Martin, C.W. (2000). Heavy Metals Trends in Floodplain Sediments and Valley Fill. Catena 39, 53-68.

- Millán, R., Carpena, R.0., Schmid, T., Sierra, M.J., Moreno, E., Peñalosa, J., Gamara, R. y Esteban, E. (2007). Rehabilitación de suelos contaminados con mercurio: Estrategias aplicables en el área de Almacén. Ecosistemas 2.

- Ministerio de Energía y Minas (MEM) (2007). Guía para el diseño de coberturas de depósitos de residuos mineros. Dirección General de Asuntos Ambientales Mineros. Sub Sector Minería, (23).

- Pineda, H. R. (2004). Presencia de hongos micorríticos arbusculares y contribución de Glomus intraradices en la absorción y translocación de zinc y cobre en girasol (Helianthus annuus L.) crecido en un suelo contaminado con residuos de mina. (Tesis doctoral). Universidad de Colima. Tecoman, Colima.

- Pollard, J., Powell, K.D., Harper, F., Smith J. (2002). The genetic basis of metal hyperaccumulation in plants. Critical Reviews in Plants Sciences, 21: 539-566.

- Porta Casanellas, J., López-Acevedo, M. y Roquero, C. (2003). Edafología para la agricultura y el medio ambiente ( $3^{\mathrm{a}}$ ed.). España: Ediciones Mundi- Prensa. 
- Prieto, J., Gonzales, C., Román, A., y Prieto, F. (2009). Contaminación y fitotoxicidad en plantas por metales pesados provenientes de suelos y agua. Redalyc. Tropical and Subtropical Agroecosystems, 10 (1), 29-44.

- Raskin, I. Y Ensley, B.D. (2000). Phytoremediation of toxic metals: using plants to clean up the environment. New York:John Wiley and Sons.

- Rodríguez 0., J.C., Rodríguez, F.H., De Lira R.G., De la Cerda, JM., Lara M., J.L. (2006). Capacidad de seis especies vegetales para acumular plomo en suelos Contaminados. Revista Fitotecnia Mexicana, 29:239-245.
- Sierra Villagrana, R. (2006). Fitorremediación de un suelo contaminado con plomo por actividad industrial. Universidad Autónoma Agraria "Antonio Narro". Buenavista, Saltillo - México.

- Spain, A. (2003). Implications of microbial heavy metals tolerance in theenvironment. Reviews in Undergraduate Research, 2, 1-6.

- Vara, M. y Oliveira, H. (2003). Metal hyperaccumulation in plants: biodiversity prospecting for phytoremediation technology. Electronic Journal of Biotechnology 6: 285-310. 